\title{
L'ARTILLERIE EN FRANCE \\ A LA FIN DU MOYEN ÂGE *
}

par

J.F. FINO

LES premiers canons, leurs fragments et les projectiles qu'ils lançaient sont fort rares aujourd'hui. Quelques uns se trouvent déposés dans les musées, tandis que d'autres existent sur la place du Marché à Gand, sur la terrasse du château d'Edimbourg, à l'entrée du Mont-Saint-Michel, etc. Toutefois, le peu de renseignements que ces objets nous transmettent, nous force d'avoir recours aux sources écrits ou figurées.

La première mention indubitable, n'offrant aucune possibilité d'interpolation ou d'erreur d'interprétation, est celle portée sur le Registro delle provvisioni de la commune de Florence. Dans les procés-verbaux des délibérations du Conseil, il est noté, à la date du 11 février 1325 (1326 n. st.) que, les prieurs, le gonfalonnier et les douze «bons hommes» sont autorisés à déléguer une ou deux personnes à la fabrication de boulets de fer et de canons de métal («palloctas ferreas et canones de metallo») pour la défense des camps et du territoire de la République ${ }^{1}$ (Fig. 1).

A la même époque, les miniatures de deux manuscrits anglais de Walter de Milmete ${ }^{2}$, nous offrent des représentations de canons primitifs. Ce sont des sortes de flacons à panse rebondie, placés, sur des tréteaux qui leurs servent d'affûts, et qui lancent des flèches (Fig. 2). Dès la première moitié du $\mathrm{XV}^{\mathrm{e}}$ siècle, peut-être même avant, les ingénieurs s'occupent, parfois longuement, des questions relatives à l'artillerie ${ }^{3}$. Tels sont l'Allemand Konrad Kyeser ${ }^{4}$ et l'Italien Marianus Jacobus, dit

* Dans les pages qui suivent artillerie signifiera exclusivement artillerie à feu.

${ }^{1}$ Archivio di Stato, Firenze. Registro delle provvisioni, vol. 22, f. 65. Publié par: L. N. Bonaparte: Études... (cf. note 11), vol. III, p. 72, note 1.

${ }^{2}$ Le De Nobilitatus Sapientiis et Prudenciis Regum, dont l'incipit de dédicace à Edouard III d'Angleterre peut être daté des environ de 1326, est conservé à la Bibliothèque du Christ-Church College, Oxford, ms. 92.

Le De Secretis Secretorum Aristotelis, lui-aussi dédié à Edouard III appartint jadis au comte de Leicester et se trouve aujourd'hui au British Museum, Add. 47,680.

${ }^{3}$ Catalogue des manuscrits d'ingénieurs de cette époque, in: Bertrand Gille: Les ingénieurs... (cf. note 12), pp. 233-239.

4 KonRad Kyeser: Bellifortis, Bibliothèque de l'Université, Göttingen, codex philosoph. 63, Édité facsimilairement, avec version allemande, par Gotz Quarg. London, Phaidon Press, 1968, 2 vols. Voir: Marcellin Berthelot: Le livre d'un ingénieur militaire à la fin du XIV siècle, in Journal des Savants, 1900, pp. 1-15 


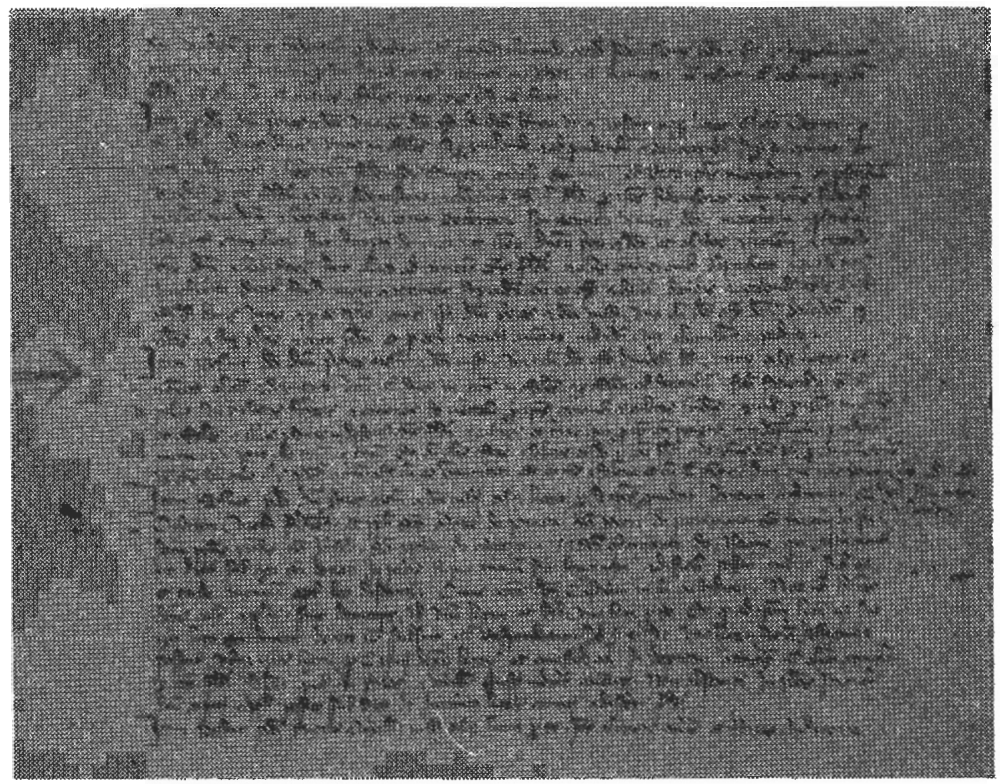

FIG. 1.-Première mention de la fabrication de canons et de boulets métalliques. Le paragraphe marqué d'une flèche indique la décision du Conseil de la Commune, figurant au vol. 22, 65 du Registro delle Provvisioni, aujourd'hui déposé à l'Archivio di Stato de Florence (Photo: Archivio).

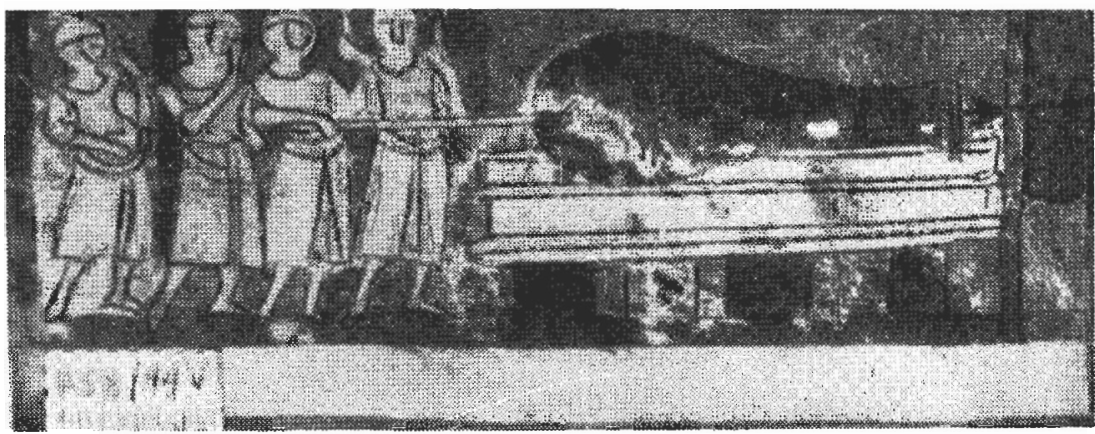

FIG. 2.-Canon anglais primitif. Miniature figurant au fol. $44 \mathrm{v}$. du De Secretis Secretorum Aristotelis de Walter de Milmete, manuscrit datant des environ de 1326 et aujourd'hui déposé au British Museum, add. 47.680. Elle représente un canon lançant une flèche; les attitudes des hommes entourant la pièce sont curieuses à observer (Photo: Courtauld Institute, London). 
Taccola, de Sienne ${ }^{5}$. Au siècle suivant, les publications se multiplient et l'on trouve, par exemple, les Italiens Vanoccio Biringuccio ${ }^{6}$ et Niccolo Fontana dit Tartaglia; ce dernier jettant les fondements de la balistique ? Mention espéciale doit être faite de Philippe de Clèves, gentilhomme bourguignon au service des rois de France, qui participerat aux guerres d'Italie sous Charles VIII et Louis XII, qui fut gouverneur de Gênes et qui rédigerat un traité de tactique ${ }^{8}$. Enfin, il y a des formulaires tout pratiques, tels le Livre du secret de l'art de l'artillerie et canonnerie ${ }^{9}$, manuscrit qui doit dater des environ de 1430 , et le Livre de canonnerie et artifices de feu ${ }^{10}$, imprimé en 1561 . Celui-ci reproduit le texte de 1430 en y ajoutant divers petits traités de dates et d'origine fort diverses où l'on trouve des formules pour raffiner le salpètre, pour fabriquer différentes sortes de poudres, des canons, des projectiles, etc.

Parmis les ouvrages modernes, il est indispensable de consulter celui de Louis-Napoléon Bonaparte, le futur Napoléon III, car, malgré une rédaction souvents confuse, il est d'un grand interêt et fait une enquête quasi-exhaustive ${ }^{11}$. Nombreux sont les travaux récents qui présentent

et 85-93. Choix de gravures reproduits par Marcellin Berthelot: Histoire des macbines de guerre et des arts mécaniques au Moyen Âge, in Annales de Cbimie et de Pbysique, 7." série, t. XIX, 1900, pp. 289-415.

${ }_{5}^{5}$ Marianus Jacobus, dit Taccola, de Sienne. Bibliothèque Nationale, latin 7239 (ms. dit «du Sérail»). Biblioteca Marciana, Venise, cod. latin XIX 5. Certaine parties figurent dans le ms. latin n. ${ }^{\circ} 197$ de Ja Bayerische Staatsbibliothek, Munich. Voir: L. Thorndike: Marianus Jacobus Taccola, in Archives Internationales d'Histoire des Sciences, t. 34, 1955, pp. 7-26. Gravures reproduites par Marcellin Ber'тнецот: Pour l'bistoire des arts mécaniques et de l'artillerie vers la fin du Moyen Âge, in Annales de Chimie et de Pbysique, 6." série, t. XXIV, 1891, pp. 433-521.

- Vanoccio Biringuccio: De la Pirolechnia libri X..., Venetia, Venturino Roffinello ad instantia di Curtio Navo, 1540, VIII, 168 f.; ibíd., La Pyrotechnie ou art du feu, trad. Jacques Vincent, Paris, C. Fremy, 1556, IV, 228 f.; ibíd., The Pirotechnia..., trad. C. Stanley Smith \& M. Teach Gundi, New York, The American Institute of Mining and Metallurgical Engineers, 1942, XXVI, $476 \mathrm{p}$.

7 Niccolo Fontana, dit Tartaglia: Nova scientia, Vinegia, Stephano da Sabio, 1537; ibíd., Quesiti e inventioni diverse, Venetia, V. Ruffinelli, 1546, 132 f.; ibíd., La Balistique, Paris, J. Corréard, 1845-1846, 2 vols.

${ }^{8}$ PhILIPPE DE Clèves: Instruction de toutes manières de guerroyer, tant par terre que par mer..., Paris, G. Morel, 1558, 151 p.

${ }^{9}$ Livre du secret de l'art de l'artillerie et canonnerie, Bibliothèque Nationale, latin 4653, ff. 125-170. Extraits, in L. N. Bonaparte: Etudes... (cf. note 11), vol. III, pp. 138-162.

in Livre de canonnerie et artifice de feut.., Paris, Vincent Sertenas, 1561, 73 f. Extraits, in J. T. Reinaud et I. FAvé: Du feu grégeois, des feux de guerre et des origines de la poudre à canon, Paris, J. Dumaine, 1845, pp. 133-169.

${ }_{11}$ L. N. Bonaparte: Etudes sur le passé el l'avenir de l'artillerie, Paris, J. Dumaine, 1846-1871, 6 vols. À partir du vol. III, rédigé par le capitaine I. Favé sur les notes de Napoléon III. 
l'histoire de l'artillerie en relation avec d'autres questions mais, nous ne mencionnerons que ceux de Bertrand Gille ${ }^{12}$, de Paul Gille ${ }^{13}$, de Philippe Contamine ${ }^{14}$, d'Edouard Perroy ${ }^{15}$ ainsi que celui de Pierre Rocolle ${ }^{16}$. D'une manière accessoire, nos Forteresses de la France médiévale ${ }^{17}$.

\section{LA POUDRE À CANON}

D'après M. Mercier, dès la seconde moitié du XII ${ }^{\mathrm{e}}$ siècle, les Arabes auraient connus la force propulsive et la force explosive de la poudre qu'ils employaient pour leurs fusées et pour leurs grenades ${ }^{18}$. Mais aucune mention n'est faite d'une troisième force, la force projective, servant à lancer un projectile au loin. Cette dernière n'apparait pas indiquée dans le Traité de combattre à cheval et des machines de guerre, véritable formulaire d'artificier rédigé en 1295 au plus tard, par l'Arabe Nedjm Eddin Hassan Alrammah ${ }^{19}$. Et, d'après le Liber ignium ad comburendos hostes,

12 Bertrand Gilie: Les ingénieurs de la Renaissance, Paris, Hermann, 1964, 239 p. Cf. c.-r. in Gladius, t. IV, 1965, pp. 113-115. Traduction anglaise par Lund Humphries, London, 1966; ibíd., Les techniques militaires, in Histoire générale des lecbriques, vol. II, Paris, Presses Universitaires de France, 1965, pp. 88-102.

${ }_{13}$ PAUL GILle: Techniques militaires [Fortifications et armement de 1500 à 1700], in Histoire gênérale des techniques, vol. II, Paris, Presses Universitaires de France, 1965, pp. 483-501.

${ }_{14} \mathrm{PH}$. Contamine: L'artillerie royale française à la veille des guerres d'Italie, in Annales de Bretagne, t. LXXI, 1964, pp. 221-261; ibíd., Guerre, Etat et Société à la fin du Moyen Âge; études sur les armées des rois de France 1337-1494, Paris, Mouton, 1972, XXXVIII, 757 pp. (Civilisations et sociétés). Cf. c.--. in Gladius, t. X, 1972, pp. $92-94$.

15 ED. PERroy: L'artillerie de Louis XI dans la campagne d'Artois, 1477, in Revue du Nord, 1943, pp. 171-196 et 293-315.

${ }_{16}$ P. Rocolle: 2000 ans de fortification française, Paris, Lavauzelle, 1973, 2 vols. Cf. c.-r. in Gladius, t. XI, 1974, p. 111.

${ }_{17}$ J. F. Finó: Forteresses de la France médiévale; construction, attaque, défense, Paris, Picard, 1967, 492 pp.; 2. édition, Paris, Picard, 1970, XIII, 510 pp. Cf. c.-r. de la 1.c édition, in Gladius, t. VI, 1967, pp. 76-88.

18 Maurice Mercier: Le feu grégeois; les feux de guerre depuis l'Antiquité, la poudre à canon, Patis, Geuthner, 1952, 164 pp. et XV pl.

19 Nedjm Eddin Hassan Alrammah (ou El Rammah): Traité de combatire à cheval et des machines de guerre, Bibliothèque Nationale, fond oriental n. ${ }^{\circ} 2825$ (ancien n..$^{\circ} 1127$, ms. à peintures); Bibliothèque Nationale, fond oriental n. 2827 (ancien fond Ancelin n. ${ }^{\circ}$ 643, ms. sans peintures); Bibliothèque Nationale, fond oriental n. ${ }^{\circ} 2829$ (ancien supplément des manuscrits arabes $\pi .0^{\circ} 733$, contient presque tout le traité de Hassan mais attribué à un autre auteur).

Echantillonage de recettes et de dessins: L. N. Bonaparte: Études... (cf. note 11), vol. III, pp. 20-24 et pl. 1-2, et J. T. Reinaud et I. FAvÉ: Du feu grégeois... (cf. note 10), pp. 20-51 et Atlas pl. I-III. 
recueil de recettes probablement d'origine byzantine mais qui ne ous est parvenu que sous sa forme latine et que l'on attribue à un tel Marchus Graechus ${ }^{20}$, cette propriété de la poudre serait inconnue dans l'Europe du XIII' siècle. Les textes parfois invoqués en vue de prouver un tel usage de cette époque sont, tous, fort sujets à caution et ce n'est qu'au début du XIV $\mathrm{X}^{\mathfrak{c}}$ que le document de Florence déjà mentionné, apporte une preuve indubitable de cet emploi.

L'origine de la poudre doit être cherchée dans le feu grégeois pulvérulent et nous en avons touché quelques mots naguère ${ }^{21}$. Les formules d'abord utilisées pour fabriquer la poudre noire souffraient d'un triple inconvénient: $1^{\circ}$ ) Faible proportion de salpètre, $30 \%$ au lieu de $75 \%$ comme il en sera au XIX ${ }^{e}$ siècle; l'adjonction de divers produits - limailles de fer, etc.- ne corrige pas ce défaut, la combustion est moins vive et le boulet, lancé majestueusement, est dépourvu de véritable pénétration. $2^{\circ}$ ) Nombreuses impuretés mêlées à ce salpètre, ce qui diminue encore la quantité de produit réellement utilisé; c'est la raison par laquelle des formulaires d'alors indiquent divers procédés visant à purifier l'indispensable ingrédient. $3^{\circ}$ ) Manque d'uniformité des grains de la poudre qui produit des effets, tantôt fusant, impropres à lancer un projectile, tantôt brisants, faisant éclater les canons. Ce n'est qu'aux environ de 1480 qu'on la soumet au grenage qui en régularise les grains, en classe les dimensions et permet d'obtenir un produit homogène dont les effets, uniformes, sont plus faciles à prévoir ${ }^{22}$.

La poudre, contenue dans des caques ou des tonneaux, est transportée sur les lieux de combat. Puis «quant une batterie est disposée, on y prélève de la poudre, qu'on met alors dans des sacs de cuir, de peau de mouton ou de toile forte. Chaque pièce a son propre $\operatorname{sac}^{23}$. Au XVI" siècle, ces sacs sont enterrés à quelque distance des canons afin d'éviter les explosions inopinées ${ }^{24}$. Il est possible que ce procédé soit déjà connu à la fin du $X V^{e}$ siècle; du moins, dès cette époque, les sacs pourris, sontils fréquemment remplacés ${ }^{25}$. La poudre est ensuite placée au fond du

${ }^{20}$ Marchus Graechus: Liber ignium ad comburendos bostes, Bibliothèque Nationale, ms. 7156 (fin du XIII siècle, début du XIV ${ }^{\mathrm{e}}$ ); Bibliothèque Nationale, ms. 7158 (XV* siècle, copie du précédent?); Bayerische Staatsbibliothek Munich, ms. 267 (similaire au 7156 de la B. N.); Bayerische Staatsbibliothek Munich, ms. 197 (écrit vers 1438, rédaction très différente).

Publié par: F. J. G. LA Porte du Theil. Paris, Delance et Lescur, 1804, VI, $18 \mathrm{pp}$., et Marcellin Berthelot: Histoire des sciences; la chimie au Moyen-Âge, vol. I, 1893, pp. 89-135, avec les variantes des ms. de Paris et de Munich.

21 J. F. FINó: Le feu el ses usages militaires, in Gladius, t. IX, 1970, pp. 15-30.

22 Paul Gille: Tecbniques militaires (cf. note 13), pp. 487.

${ }^{23}$ Bibliothèque Nationale, français 7881, f. 92 v., f. 153 v., p. 162.

${ }^{24}$ Ibid., 388.

${ }^{25}$ Ibid., 7881, f. 154. 
tube au moyen d'un instrument appelé chargoner, composé d'une hampe en bois, pour laquelle on utilise un fût de pique ou de javeline, et d'un repoussoir. Ce repoussoir est en cuivre, en bois ou en bronze; il s'agit, semble-t-il, d'une sorte de pelle allongée dans laquelle on met la poudre et qu'on retourne en fin de course ${ }^{26}$. Naturellement qu'après chaque coup, la pièce est nettoyée intérieurement à l'aide d'un écouvillon, brosse cylindrique montée sur un long manche, débarrassée des grains de poudre non entièrement éteints, etc. mais, malgré ces précautions, des départs intempestifs se produisent souvent.

\section{LES PIÈCES}

Les canons ont été construits avec des matériaux fort diverses. Au Musée de l'Armée (Galerie de l'Orient, cote N 708) on peut voir un canon conchinchinois, pris en 1862, formé d'une tôle de fer enrobée de bois. La pièce, longue de $1,90 \mathrm{~m}$., de $0,097 \mathrm{~m}$. de calibre, est très primitive ${ }^{27}$ et, peut-être, les canons représentés dans les manuscrits de Walter de Milmete (Fig. 2) étaient-ils faits d'une manière analogue.

Les pièces formées de barres de fer forgé, juxtaposées comme les douves d'un tonneau et serties par de anneaux, de fer aussi, ont dû apparaïtre en Europe de très bonne heure. En 1375, pour le siège du château de Saint-Sauveur-le-Vicomte, le roi de France Charles V fait fabriquer à Rouen plusieurs canons, dont le plus grand nécéssite $1.000 \mathrm{~kg}$. de fer et $100 \mathrm{~kg}$. d'acier ainsi que le travail de cinq maïtres forgerons et de leurs aides pendant 45 jours ${ }^{28}$.

Le désir d'utiliser un métal moins lourd pousse les Italiens à employer le cuivre, matériau qui ne leur donne pas entière satisfaction. Pourtant, dans le premier quart du XVII ${ }^{\mathrm{e}}$ siècle, Gustave-Adolphe de Suède a des canons formés par un mince tube de cuivre, entouré de douves en bois fortement ficelées et recouvertes d'une enveloppe de cuir; ces canons, ne pesant qu'une soixantaine de kilos, placés sur des affûts à grandes roues et attelés de deux chevaux, forment une véritable artillerie légère, ce qui explique, en partie, les succés suèdois au début de la guerre de Trente ans.

Les canons sont faits parfois en fonte de fer malgré la fragilité du produit primitivement livré par les hauts-fourneaux. Mais le bronze

${ }^{26}$ Ph. Contamine: L'artillerie royale... (cf. note 14), p. 249.

${ }^{27}$ Précisions aimablement communiquées par le Colonnel Maurice Houriez.

${ }^{28}$ Archives Nationales, registre cote KK 350, f. 254 v. 264 v. Publié par: L. N. Bonaparte: Éludes... (cf. note 11), vol. IV, pp. XVIII-XXXVI. 
s'avère être le meilleur matériau. Les fondeurs français trouvent la juste proportion d'étain ( 8 à $12 \%$ ) qui donne à l'alliage la dureté requise et l'artillerie de Louis XI est de bronze. Il en sera de même pendant des siècles et encore en 1914, la plupart des canons de l'armée italienne seront de bronze, spécialement durci il est vrai. On coule les pièces en suivant un procédé analogue à celui employé pour fabriquer les cloches; c'est l'époque où renaît la statuaire de métal et les grands artistes d'alors ne dédaignent pas de consacrer leurs efforts à la décoration des pièces d'artillerie. On verse le métal en fusion dans un moule en forme de cylindre creux dont l'axe est occupé par un noyau qui donne naissance à l'âme de la pièce ${ }^{29}$; naturellement que la préparation du moule, la conduite et l'homogénéité de la coulée, etc., soulèvent nombre de difficultés mais, malgré cela, le procédé s’impose. La régularité de l'âme est parachevée par l'alésage au moyen de machines qui, dès le milieu du $\mathrm{XV}^{\mathrm{0}}$ siècle apparaissent représentées dans les carnets d'ingénieurs.

Toutefois, le fer ne perd pas ses droits. Dans le dernier quart du $\mathrm{XV}^{\mathrm{e}}$ siècle, fer forgé et bronze coexistent dans l'artillerie du duc de Bourgogne Charles le Téméraire ${ }^{30}$. Pendant la première moitié du XIX Xen- $^{\mathrm{e}}$ core, la plupart des canons armant les navires sont de fonte de fer et P. Gille fait remarquer avec raison que les canons de bronze sont souvent appelés «de fonte verte» ou «de fonte» tout court, tandis que ceux de fonte de fer (et non plus de fer forgé) sont dits «de fer» ${ }^{31}$.

Notons incidemment que, fort souvent, le matériau provenant de pièces hors d'usage est employé à en fabriquer de nouvelles. C'est ce que montre, entre autres, un document datant des environ de 1472 et étudié par R. Fawtier où il est dit que deux gros canons, trois serpentines, une bombarde, etc., de fonte, ont été employés à couler, à Orléans, une grosse bombarde nommée La Réale ${ }^{32}$.

Les inventaires de matériel déposé à la Bastille et à l'Hôtel-de-Ville de Paris ${ }^{33}$, les comptes des duc de Bourgogne ${ }^{34}$, etc., permettent d'esquis-

${ }^{29}$ L'opération est décrite d'une façon sommaire, in Histoire générale des techniques, vol. II (cf. note 12), pp. 60-61 et 484-485.

${ }^{31}$ Fl. Deuchler: Die Burgunderbeute; inventar der Beutesiücke aus den Schlachten Von Grandson, Murten und Nancy, 1476-1477, Berne, Stämpli, 1963, XXII, 418 pp. Cf. c.-r. in Gladius, t. VI, 1967, pp. 75-76.

${ }^{31}$ PaUl Gille: Techniques militaires (cf. note 13), pp. 493-494.

32 John Rylands Library, Manchester, ms. French 71, f. 7. Etudié par: R. FAWTIER: Documents inédits sur l'organisation de l'artillerie royale au temps de Louis XI, in Essay... to Th. F. Tout, Manchester, 1925, p. 374.

${ }^{33}$ Archives Nationales, section ancienne, P. 1189 , pièces $X V^{2}, X V$ et 16 ; íd., íd., K 952, n. 3 (liasse); Bibliothèque Nationale, ms. français 6970, ff. 392-394. Publié par: L. N. Bonaparte: Éludes... (cf. note 11), vol. I, pp. 366-383.

${ }^{34}$ Resumés par: L. N. Bonaparte: Ėtudes... (cf. note 11), vol. III, pp. 128-137. 
ser un classement des pièces en usage au $X V^{e}$ siècle. On trouve d'abord les grands canons ou grosses bombardes qui pèsent jusqu'à $15.000 \mathrm{~kg}$. et lancent des boulets d'un poids atteignant les $450 \mathrm{~kg}$. Les courtaux, crapauds ou gros bastons, sont des sortes de bombardes, moins lourds que celles-ci. Larges et ramassés, ils figurent encore en 1477 parmis l'artillerie mobile de Louis XI mais, en 1494, ils ont disparus de celle de Charles VIII; au XVI ${ }^{\mathrm{e}}$ siècle, ils seront affectés à la seule défense des places. Les veuglaires, qui subsisterons jusqu'à la fin du $X V^{\mathbf{e}}$ siècle et parfois au-delà, sont des tubes plus longs et plus minces que les bombardes. Ils pèsent de 150 à $5.000 \mathrm{~kg}$. et lancent des boulets de 1,50 à $50 \mathrm{~kg}$. L'artillerie légère se compose de crapodeaux et de couleuvres ou coulevrines, d'un poids de 6 à $25 \mathrm{~kg}$., lançant des projectiles d'environ $1 \mathrm{~kg}$. Enfin, des mortiers sont parfois utilisés pour le tir vertical. Lorsque plusieurs petites pièces sont réunies et montées sur un chassis de bois pourvu de roues, protégé par un mantelet et muni d'un coffre à munitions, on a les orgues ou ribaudequins, permettant de déplacer une puissance de feu assez considérable.

Dans le troisième quart du XV $\mathrm{XV}^{\mathrm{e}}$ siècle, Francesco di Giorgio Martini, célèbre architecte et ingénieur italien, avait proposé de réduire à dix les types de canons utilisés ${ }^{35}$. Son opinion ne fut pas suivie et vers 1525 , Tartaglia en énumère vingt-six couramment employés par les armées péninsulaires. Une telle diversité entraîne toute sortes d'inconvénients et rend difficile la fabrication en série des boulets. On s'efforce donc de la réduire et, entre 1512 et 1530 , Charles-Quint régle ce qui concerne la fabrication et les essais des canons armants ses troupes ainsi que leurs calibre. Il limite le nombre de ceux-ci et les détermine par le poids du boulet qu'ils lancent. On a donc des canons de 40, de 24, de 12, de 61 / 2 et de 3 livres. Son adversaire, François I. ${ }^{\text {er }}$ réduit aussi le nombre de calibres utilisés: le double canon, le canon serpentin, la grande coulevrine, la bâtarde, la moyenne et le faucon. Sous son fils Henri II, on élimine le double canon et c'est vraisemblablement par une ordonnance royale de 1544 - Jean d'Estrées étant Grand Maître de l'artillerie- que les «six calibres de France» s'imposent. Le tableau suivant en présente les principales caractéristiques ${ }^{36}$.

35 Bertrand Gille: Les techniques militaires (cf. note 12), p. 94.

${ }^{36}$ L. N. Bonaparte: Études... (cf. note 11), vol. III, pp. 237, 241, 243 et 251. 


\begin{tabular}{|c|c|c|c|c|c|}
\hline \multirow{2}{*}{ DÉNOMINATION } & \multirow{2}{*}{$\begin{array}{l}\text { POIDS DE } \\
\text { LA PIËCE } \\
\text { (livres) }\end{array}$} & \multicolumn{2}{|c|}{ CALIBRE } & \multirow{2}{*}{$\begin{array}{l}\text { POIDS DU } \\
\text { PROIECTILE } \\
\text { (livres) }\end{array}$} & \multirow{2}{*}{$\begin{array}{l}\text { ATTELAGE } \\
\text { (chevaux) }\end{array}$} \\
\hline & & (pouces) & (lignes) & & \\
\hline Canon...... & 5.400 & 6 & 3 & 33 & 21 \\
\hline Grande coulevrine .......... & 3.800 & 4 & 4 & 16 & 17 \\
\hline Coulevrine bâtarde ........ & 1.970 & 3 & 10 & 8 & 11 \\
\hline Coulevrine moyenne ...... & 870 & 2 & 9 & 3 & 7 \\
\hline Faucon $\ldots \ldots \ldots \ldots \ldots \ldots \ldots$ & 750 & 2 & 4 & 2 & 4 \\
\hline Fauconneau $\ldots \ldots \ldots \ldots \ldots$ & 450 & 1 & 10 & 1 & 2 \\
\hline
\end{tabular}

Primitivement, les veuglaires et les calibres au-dessous se chargent par la culasse. La pièce se présente sous la forme d'un tube ouvert aux deux extrémités. Une boîte de fer, contenant la charge de poudre et un tampon, est appliquée contre l'extrémité postérieure du tube, où l'on a déjà placé le projectile. La boîte est retenue par une sorte d'étrier, en fer aussi, fixé à l'un des anneaux sertissant la pièce et qui pivote latéralement. Cet étrier se détraquant avec facilité, on fait bientôt appel à un autre système. L'extrémité postérieure du tube est fermée d'une façon définitive mais on ménage, sur le côté, une ouverture par laquelle on introduit la boîte; des chevilles et des coins de bois ou de métal servent à l'ajuster contre le boulet et l'âme du tube (Fig. 3). L'usage des boîtes préparées d'avance permet d'assurer un tir relativement rapide et ces pièces sont souvent placées, en soutien, à côté des bombardes dont la manoeuvre est plus lente. Lorsque l'arme est de petit calibre, elle peut être articulée sur une sorte de fourche, munie d'une tige servant de pivot, qui pénètre librement dans un socle de bois ou dans la maçonnerie de la muraille; la culasse êtant prolongée par un bras d'une cinquantaine de centimètres de long, il est aisé de braquer le canon dans n'importe quelle direction. De belle collections d'armes de ce genre sont conservées au Musée de la Porte de Hal (Bruxelles) et à celui de l'Armée (Paris). Ce dernier en expose plusieurs, notament sous les cotes $\mathrm{N} 5$ à $\mathrm{N} 7$, Galerie de l'Orient, qui datent de la fin du XIV siècle ou du début du $\mathrm{XV}^{\mathrm{c}}$; elles sont formées de douves soudées entre-elles et serties de frettes. Sous les cotes N 24 à N 30, on trouve des pièces du milieu du XV', fabriquées d'une plaque de fer, recourbée et soudée parallèlement à l'axe.

Cependant la jonction boîte-âme étant imparfaite, il se produit des fuites de gaz, d'où déperdition de forces et égueulement des bords, ce qui fait abandonner le système. Le canon classique a sa culasse entièrment fermée et on le charge par la gueule. Après avoir nettoyé l'intérieur, on introduit, successivement, la poudre, un tampon isolant, le projectile 

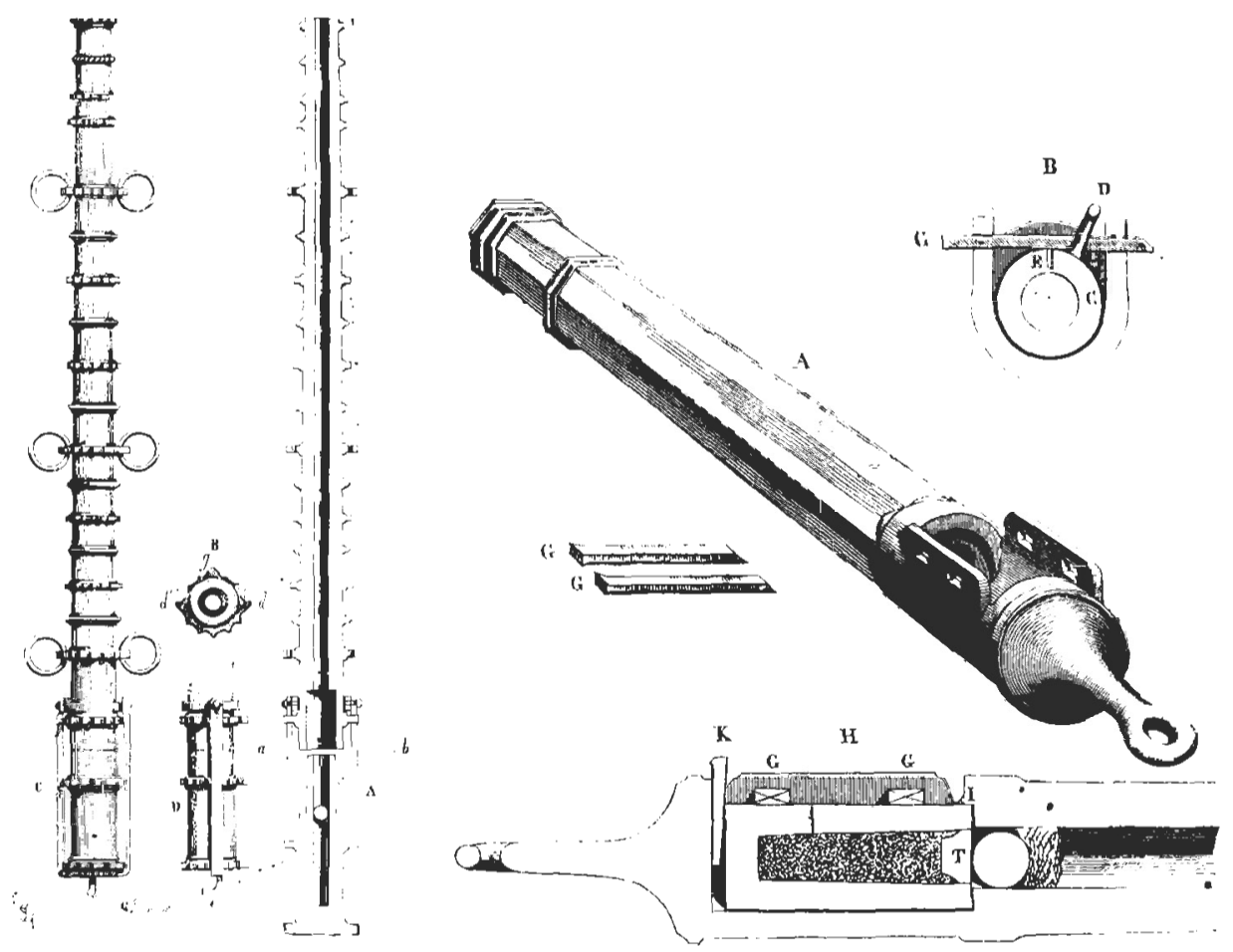

F1G. 3.-Canons se chargeant par la culasse. A gauche, dessin d'une pièce dite «à étrier mobile»; les anneaux sertissant le tube servaient, probablement, à le fixer sur un tréteau faisant fonction d'affût. A droite, dessin d'une autre, dite «à encastrement»; les clavettes $G$ serrent la boîte chargée contrc la paroi inférieure de l'encastrement, tandis que le coin $K$ la repousse contre l'orifice de l'âme où se trouve le projectile (E. Viollet-le-Duc: Dict. architéct., vol. 5, pp. 247 et 253).

et la bourre. Ce n'est que dans la seconde moitié du XIX ${ }^{\mathrm{e}}$ siècle que les progrés des industries mécaniques permettront de revenir aux armes se chargeant par la culasse.

Les tubes sont, généralement, cylindriques mais, aux $\mathrm{XV}^{e}$ et $\mathrm{XVI}^{\mathbf{c}}$ siècles, on peut leur donner une forme extérieure polygonale, à huit pans. A cause de leurs poids, le transport des grosses bombardes s'avère difficile; on les divise alors en chambre et en volée. La chambre, où l'on place la poudre, est formée de lamelles de fer soudées entre-elles. La volée, c'est-à-dire le canon proprement dit, peut se diviser, à son tour, 
en plusieurs tronçons. Chaque partie est munie de filetages et d'encastrements pour les leviers, ce qui permet un montage adéquat (Fig. 4). Enfin, il faut signaler que certaines coulevrines sont formées d'un tube assez court (moins de 1,50 m.) ajusté à une queue de bois, lourde, massive, longe d'environ $2 \mathrm{~m}$., qui sert de crosse et de contrepoids; pour

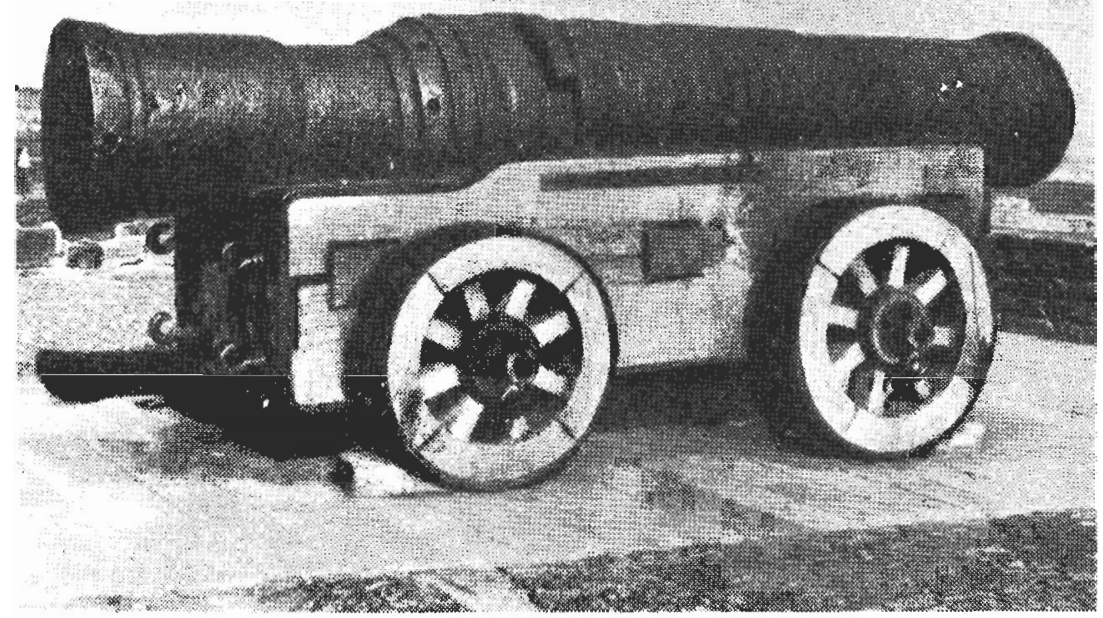

Fig. 4,-La Mons Meg d'Edimbourg. La pièce, conservée sur la terrasse du château, est posée sur un affût reconstitué d'après un ancien bas-relief existant au château même. $\grave{A}$ gauche, la chambre servant à contenir la charge de poudre. $A$ droite, la voléc ou canon proprement dit. On apperçoit nettement les cavités destinées à recevoir les leviers vissant chambre et volée (Photo: S. Domicellj).

le tir l'extrémité de la crosse est fichée dans le sol, tandis que le canon est relevé au moyen d'une fourche plantée à l'avant. Divers payements de sommes dues pour les gages des «joueurs de coulevrines» et de leurs aides, pour l'achat de projectiles ou de matériaux, etc., sont ainsi portés sur les comptes, notament sur ceux relatifs au siège d'Orléans en $1428{ }^{37}$.

${ }^{37}$ Journal du siège d'Orléans, 1428-1429, augmenté... des comptes de ville 1429-1431... [et des comptes de forteresse]; éd. P. Charpentier et Ch. Cuissard. Orléans, H. Herluison, 1896, LVI, 410 pp. Voir pp. 255, 256, 275, 286, 300, etc. 
Ces coulevrines semi-portatives (Fig. 5), quoique appartenant à l'artillerie légère, constituent un antécédent des bâlons à feu, des bacquebuttes, des arquebuses, etc., qui s'imposeront au $\mathrm{XVI}^{\mathrm{c}}$ siècle, et l'arme à feu individuelle dérive du canon et non à l'inverse, ce qui semblerait plus logique.
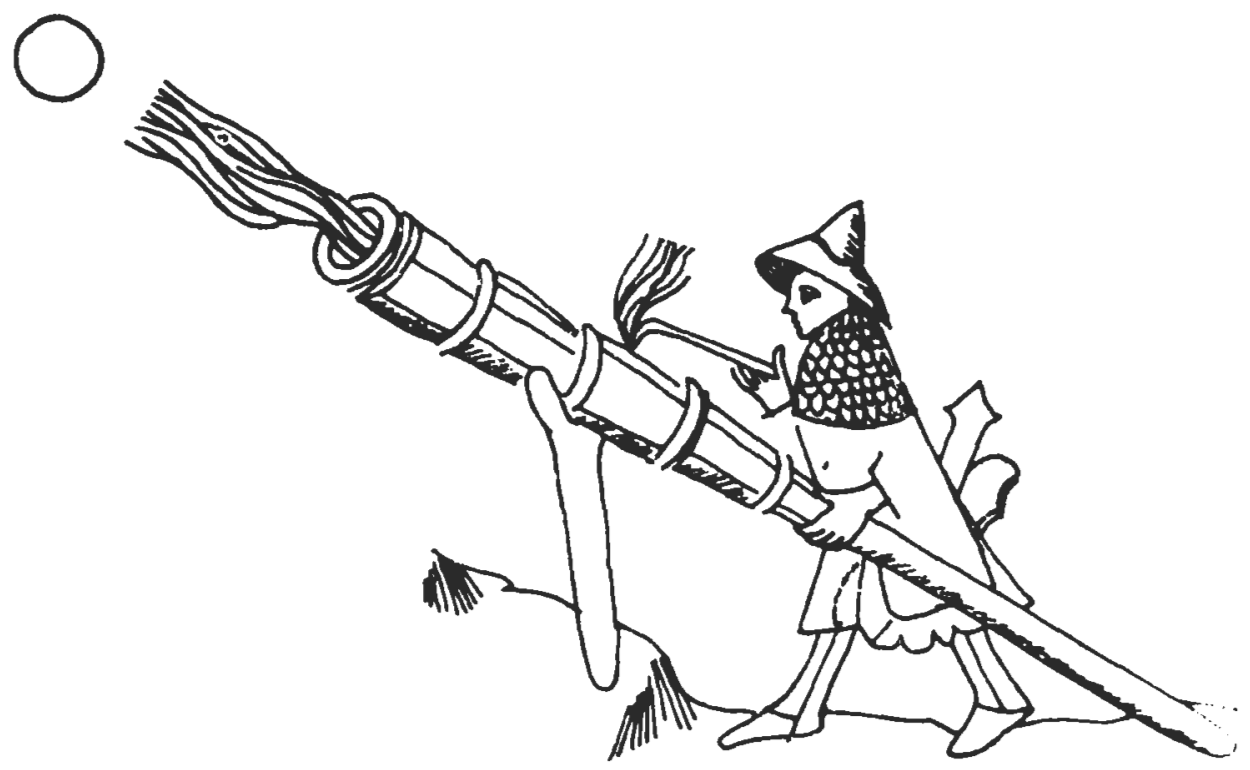

FIg. 5.-Coulevrinier vers 1400. L'arme, posée sur une fourche, lance un boulet. D'après le Bellifortis de Konrad Kyeser, manuscrit aujourd'hui déposé à la Bibliot. Universit. de Göttingen, codex philosoph. 63, fol. 104 v. (Photo: Bibliothek.).

\section{LES PROJECTILES}

Au début, les pièces anglaises et françaises - toutes de petit calibre cela s'entend- lancent des sortes de flèches ou garrots de métal. À la Tour de Londres, sous la cote XX 301 on conserve un de ces trait paraissant dater du XVI ${ }^{\mathrm{e}}$ siècle; c'est une tige de métal, longue de $0,35 \mathrm{~m}$., dont une extrémité est armée d'une pointe à double crochet, tandis que l'autre, aplatie à fin de servir d'empennage, se termine par un pas de vis où l'on fixe une bourre de bois s'ajustant à l'âme de l'arme. Le projectile figuré dans les manuscrits de Walter de Milmete est fort semblable (Fig. 2). Par contre, les canons italiens lancent des boulets et à en juger par le document de Florence (Fig. 1), déjà en 1325-1326 ces boulets 
étaient de métal («palloctas ferreas» dit le texte). Pour rendre plus efficace le tir contre des obstacles résistants, Anglais et Français adopteront eux-aussi le boulet métallique mais il ne le feront pas d'un seul coup. D'abord, ils se servent de boulets de pierre similaires à ceux des anciens engins, trébuchets et mangonneaux ${ }^{38}$, ce qui conduit à employer des bombardes d'un calibre de 0,50 mt. et plus encore. Puis, pour accroitre la masse et la résistance au choc de ces projectiles, on les cercles ou on les chape de métal. Enfin, dans le troisième quart du $\mathrm{XV}^{\mathrm{e}}$ siècle on adopte le boulet de fonte, matériau que les métallurgistes produisent désormais de façon assez abondante. C'est la révolution dite «du boulet métallique» qui, d'abord limitée aux pièces de petit calibre, aura de nombreuses conséquences. Pour nous en tenir à ce qui a trait à l'artillerie, on notera qu'en premier lieu, les projectiles, beaucoup plus résistant, n'éclatent pas sous l'impact. D'autre part, la fonte étant environ trois fois plus dense que la pierre, le volume du boulet de fonte est bien moindre que celui du boulet de pierre de même poids. Si le calibre de ce dernier est de $0,50 \mathrm{~m}$. (cas du canon d'Edimbourg) celui du boulet de fonte n'est que de $0,3464 \mathrm{~m}$. Or, en négligeant divers facteurs, tels les tourbillons qui se produisent autour du projectile, une charge de poudre qui imprime une vitesse égale à 1 à un boulet de pierre, imprimera une vitesse de 2,08 à un autre de fonte de poids égal. La force vive d'un projectile étant donnée par la formule $f=m v^{2}$ ( $m$ est le poids du boulet et $v$ sa vitesse), il s'ensuit que celle du boulet de fonte est sensiblement le quadruple $-(2,08)^{2}=4,33$ - de celle du boulet de pierre. Inversemment, si les forces vives des deux boulets sont égales entre elles, le diamètre du boulet de fonte est environ le tiers de celui du boulet de pier$\mathrm{re}^{39}$. On peut donc obtenir des effects destructeurs identiques avec des canons de calibre bien plus petit, ce qui entraine l'abandon des très grosses pièces, lesquelles ne réapparaitrons que dans le dernier quart du XIX ${ }^{c}$ siècle et ce jusqu'à la guerre de 1914-1918 (canons allemands de $0,42 \mathrm{~m}$. et français de $0,52 \mathrm{~m}$.).

Les coulevrines semi-portatives ainsi que certains faucons et fauconneaux, lancent des plombées ou plommées (balles sphériques de plomb) ou des margots (balles cylindriques) ${ }^{40}$. Le métal, fondu dans une sorte de louche de fer, est ensuite versé dans des moules de cuivre ou de fonte que l'on tient au moyen de fortes tenailles. Pour accroitre le poids

${ }^{38}$ V. GAY: Glossaire archéologique du Moyen Âge el de la Renaissance, Paris, Picard, 1887-1928, vol. I, p. 190, art. boulet.

${ }^{39}$ Nous remercions $M$. Michel Herzen, architecte à Lausanne, de nous avoir fourni ces précisions d'ordre balistique.

${ }_{40} \mathrm{~V}$. GaY: Glossaire archéologique ( $\mathrm{cf}$. note 38); articles margot et plommée (vol. II, pp. 115 et 241 ). 
de la balle, il arrive souvent que l'on place, au centre, un bloqueau de fer.

Il est évident qu'on ne se borne pas uniquement à utiliser l'impact des projectiles mais que l'on tâche aussi d'employer ceux-ci à répandre les feux de guerre. Dès 1356, au siège du château de Breteuil, un gros et fort beffroi construit par les assaillants, est rapidement mis hors d'usage par les défenseurs au moyen des projectiles incendiaires de leur artillerie ${ }^{41}$. Des pots-à-feu ainsi lancés apparaissent figurés dans le livre de Taccola qui doit dater de la première moitié du $X V^{e}$ siècle ${ }^{42}$ et, à la fin du $X V^{\mathrm{e}}$, début du XVIc, plusieurs traités militaires montrent des bombes faites de deux hémisphères creux, en fer battu, remplis de produits incendiaires ou explosifs, que réunissent des brides ou des frettes ${ }^{43}$.

\section{AFFÛTS ET ACCESSOIRES}

Au début, les canons semblent avoir été fixés sur des caisses de bois et le canon conchinchinois du Musée de l'Armée est ainsi assujetti. On les transporte à dos d'animal ou sur des chariots (l'avanttrain mobile, organe essentiel d'une bonne voiture, semble se répandre en Europe au $\mathrm{XIV}^{\mathrm{e}}$ siècle). Arrivé sur place, on débarque les caises, on les entoure de planches pour limiter les dégats dans le cas où les tubes éclateraient, et on les affûte, c'est-à-dire qu'au moyen de cales et de leviers, on les oriente et on leurs donne l'inclinaison désirée. Plus tard, en vue d'accroitre la rapidité de la mise en batterie, on monte les pièces directement sur des roues; grâce au grenage de la poudre, les éclatements intempestifs étant moins à craindre maintenant. Pour faciliter le pointage, on place les canons sur de véritables affûts, divisés en deux éléments de bois. L'élément du-dessus, auquel est fixé le tube, s'articule sur le second, portant les roues. Une crémaillère permet alors au premier tronçon de prendre l'angle d'inclinaison voulu. Des affûts de cette espèce pris sur les Bourguignons à la bataille de Grandson (1476), sont visibles au Musée suisse de La Neuveville (Fig. 6).

La mise à feu a lieu en approchant une broche métallique, rougie au feu, d'une petite ouverture, la lumière, forée dans le métal de la boîte ou de la chambre; la broche est, naturellement, fort longue comme pré-

${ }^{41}$ Froissart: Chroniques, Liy. I, $\$ 369$; éd. S. Luce, G. Raynaud et L. Mirot, Paris, Société de l'Histoire de France, 1869 et sqq., vol. IV, pp. 193-196.

${ }^{42}$ Marcellin Berthelot: Pour l'bistoire des arts mécaniques (cf. note 5), pp. $473-474$.

${ }^{43}$ E. Violnet-Le-Duc: Dictionnaire raisonné de l'architecture française du XI au XVIe siècle, Paris, Bance, 1858-1868, vol. V, p. 251. 
caution supplémentaire contre l'éclatement des pièces. Mais, malgré que l'on ait soin de l'arroser d'eau pour la raffraichir, la lumière s'élargit très vite ( 60 ou 70 coups) et le canon devient inutilisable. Vers 15501561, on a l'idée de percer la lumière dans un petit morceau de fer ou d'acier introduit à la place adéquate. L'idée est bonne mais ces nouvelles lumières se rouillent facilement.

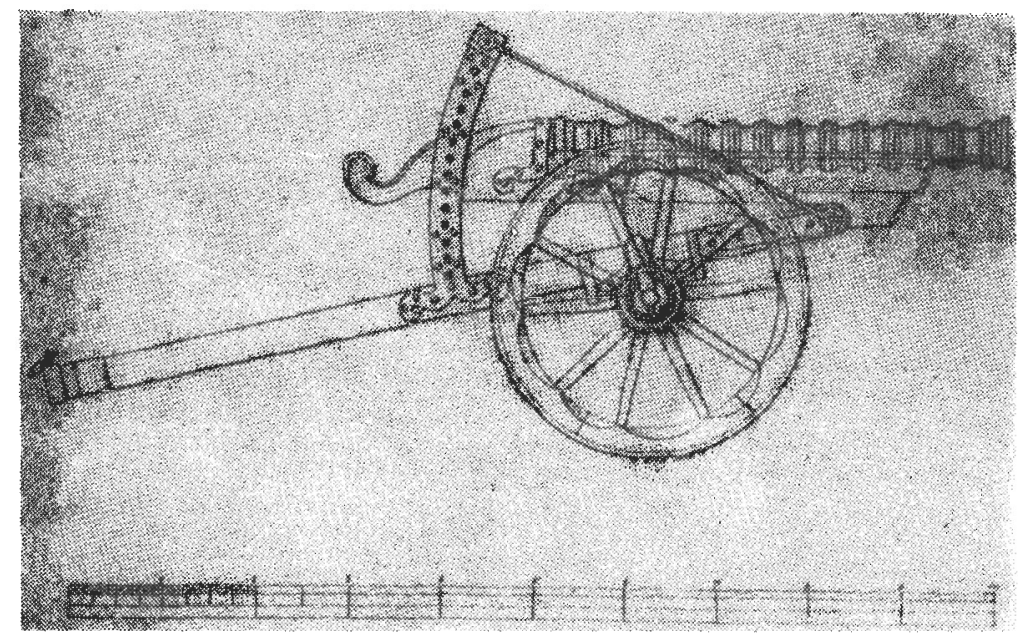

FIG. 6.-Canon de campagne de la deuxième moitié du XVe siècle. Dessin d'après une pièce bourguignonne prise par les Suisses à la bataille de Grandson et qui se trouve au Musée de La Neuveville. Construite en fer forgé, aux environ de 1460 , elle a $0,75 \mathrm{~m}$. de diamètre intérieur et lançait des boulets métalliques. On voit, parfaitement, l'articulation des deux éléments formant l'affût ainsi que la crémaillère permettant de maintenir le tube à l'angle d'inclinaison voulu (FL. Deuchler: Die Burgunderbeute, p. 314).

Pour mesurer l'inclinaison de la pièce, on se sert de l'équerre de canonnier. C'est un long bâton que l'on couche sur l'arête inférieure de l'âme et qui porte, à son extrémité, un index lesté qui se déplace contre un quart de cercle gradué. À la Tour de Londres, sous le nom de «gun. ner's quadrant», cote XIV 19, on conserve un de ces objets, richement orné, exécuté en 1585 pour le prince Jules de Brunswick-Lunebourg et un basrelief du château d'Edimbourg montre le pointage de la Mons Meg au moyen d'un instrument analogue.

Aux environ de 1460 , on commence à utiliser les tourillons, petits cylindres de métal faisant saillie de chaque côté du canon, vers le milieu 
de sa longueur. Ces tourillons, d'abord faits d'un morceau rapporté sont, ensuite, coulés en même temps que le tube. Ils permettent à celui-ci de basculer sur un plan vertical et plus n'est besoin d'un affût articulé. Les canons de l'armée de Charles VIII apparaissent munis de ce dispositif qui, plus ou moins perfectionné, subsiste encore.

Les anses, connues des Allemands depuis longtemps ${ }^{44}$, permettent de manoeuvrer les tubes sans avoir besoin de les entourer de cordages, de cables, etc.

Lors du siège et de la défense d'une place, les pièces tirent à travers des ouvertures pratiquées dans les murailles. Celles-ci peuvent être soit de maçonnerie, soit de terre contenue dans des futailles ou des gabions, sortes de paniers d'osier qui semblent apparaître vers la fin du XV' siècle. Pour protéger du feu ennemi les servants occupés au chargement, de lourds volets de bois se rabattent sur l'ouverture. Dans certains cas, ces volets, fixés aux pièces elles-mêmes, constituent de véritables boucliers, analogues à ceux des canons contemporains.

Naturellement, de nombreuses plaques de fer renforcent les affuts et, en vue de faciliter les déplacements, Jacques Galiot - maître, visiteur et réformateur de l'artillerie sous Louis XI- aurait inventé l'escuage ou écuanteur de roues en faisant que leurs rayons ne soient pas perpendiculaires à l'axe mais forment un angle aîgu avec celuici ${ }^{45}$.

\section{LE TIR}

La portée et les effects de l'artillerie, très faibles au début ce qui explique pourquoi on ne s'admire guère lors de la naissance de celle-ci n'augmenteront que fort lentement. En 1347, Hugues de Cardaillac rend une ordonnance sur le façon dont la garnison du château de Bioule le doit être disposée pour la défense. Dans l'art. 14, il est dit que face à une attaque, les assiègés «tirent tout d'abord avec les arbalètes à tour qui portent plus loin et, après, avec les arbalètes à deux pieds, et avec les pierres et les canons, de la manière qu'ils jugeront le plus convenable pour la défense» ${ }^{46}$. De 1356 à 1380, Sicard de Lordat, sur l'ordre de son maître Gaston Fébus (ou Phébus), couvre le Béarn d'un réseau de fortifications dans lesquelles il ne tient aucun compte de l'usage du

* Paul Gille: Techniques militaires (ct, note 13), p. 490.

${ }^{45}$ Ibíd., p. 488.

${ }^{40}$ Original disparu lors de l'incendie du château de Bioule. Publié par: L. N. BoNAPARTE: Études... (cf. note 11), vol. IV, pp. IX-XIV. Edition révisée: E. ForesTIÉ: Hugues de Cardaillac et la poudre à canon, in Société archéologique du Tarnet-Garonne. Bulletin, t. 29, 1901, pp. 99-132, 185-222 et 297-312. Voir pp. 203-216. 
canon, bien qu'il fut le chef de l'artillerie comtale ... Le château de Pierrefonds, bâti au début du $X^{\mathrm{e}}$ siècle et le nouveau château de Langeais, construit par ordre royal en 1465-1467, ne sont conçus qu'en fonction des armes médiévales. En 1468-1473, Jean Bourré —qui avait été Capitaine de Langeais et qui fut homme de confiance de Louis XI ainsi que de Charles VIII et de Louis XII- fait bâtir son château du Plessis-Bourré, près d'Angers. Les très larges douves qui entourent le château de toutes parts suffissent à le protéger contre les effets de l'artillerie. A l'époque de Louis XIV, en plein $\mathrm{XVII}^{\mathrm{e}}$ siècle, les canons d'une place n'ont guère qu'une portée utile de $500 \mathrm{~m}$.

Le tir manque de justesse, ne serais-ce que par le vent, intervalle existant entre le boulet et l'âme de la pièce. On essaye bien de le diminuer par divers artifices et de s'entrainer par des exercises de pointage tels ceux auxquels participe Charles VIII à Moncalieri, près Turin ${ }^{47}$, mais la question ne serat vraiment résolue que par l'adoption de l'artillerie rayée, qui aurat lieu dans la seconde moitié du XIX ${ }^{\mathrm{c}}$ siècle et qui permettra de superposer les coups succesifs.

Par contre, la cadence du tir s'accroit rapidement. Lors du siège de 1453, chacun des gros canons de Mahomet II qui battaient les murailles de Constantinople, d'un calibre d'environ $0,75 \mathrm{~m}$. et lançant un boulet de pierre de plus de $580 \mathrm{~kg}$, ne tiraient guère que sept fois pendant le jour et une seule fois pendant la nuit, vu le temps requis par l'affutage de l'arme après chaque coup. A la fin de ce même siècle, grâce aux divers perfectionnements dont elle a été l'objet, l'artillerie française tire de 30 à 40 coups par pièce et par jour ${ }^{48}$. D'autre part, les progrès de la métallurgie permettront d'augmenter sensiblement de nombre de pièces et celui des boulets. Déjà à Marignan (1515) François $\mathrm{I}^{\mathrm{er}}$ met en ligne 72 gros canons et «on pourrait sans doute estimer les besoins de la seule artillerie de Charles-Quint, au cours de l'année cruciale 1552-1553, à plus de 200.000 boulets; au siège de Magdebourg en 1631, Tilly réduira la ville par des bombardements journaliers de quelques 12.000 à 18.000 coups pendant un mois» ${ }^{49}$.

${ }^{47}$ Fr. VAux de Folet1er: Galiot de Genouillac, maître de l'artillerie de France, 1465-1546, Paris, Picard, 1925, p. 29.

4+ Ph. Contamine: L'artillerie royale française... (cf. note 14), pp. 247, note 99.

19. J. SCHNEIDER: Fer et sidérurgie dans l'économie européenne du XI au XVII siècles, in Colloque international "Le fer à travers les âges», Nancy, 3-6 octobre 1955. Actes, Nancy, Soc. d'impression typographique, 1956 (Annales de l'Est, Mémoire n. 16), p. 132. 


\section{L'ORGANISATION}

L'artillerie naissante semble s'être développée surtout dans les grosses communes de Flandre, du Nord de la France et de l'Italie, Peut-être cela est-il dû au fait que ces villes ćtaient les seules à posséder les ressources financières et techniques que l'artillerie exigeait; en cas de besoin on se la prêtait réciproquement et les exemples d'une telle opération abondent. Mais on assiste vite à une ample diffusion de l'arme nouvelle et sous la direction de Picrre Bessoneau (1420-1440) puis de ses successeurs les frères Bureau ${ }^{50}$, l'artillerie royale fait de sensibles progrès. Sous ces derniers se place, notamment, la généralisation du boulet métallique et l'adoption d'affûts plus maniables. Charles VII dispose ainsi d'un matériel nombreux, qui fait l'admiration de ses contemporains et qui s'avère hautement efficace. A la fin de la guerre de Cent ans, lors de la campagne de Normandie, en 1450, ses troupes reprènent en un an et une semaine une soixantaine de places; or la conquête de chacune d'elles avait coûté aux Anglais de longs mois de siège. En 1453, à la bataille de Castillon, près de Libourne, en Dordogne, les Anglais - pourtant commandés par le célèbre Talbot - sont littéralement fauchés par le feu des Français.

A la fin du XVe siècle, l'artillerie royale est placée sous les ordres du Grand maître de l'artillerie et distribuée en «bandes». Chaque bande, commandé par un capitaine, comprend une trentaine ou une quarantaine de pièces de différents calibres, servies par un millier d'hommes. Ceux-ci se distribuent en «personnel ordinaire» (officiers, contrôleurs financiers, fondeurs et canonniers ordinaires, forgerons, etc.) peu nombreux mais quasi permanent, et «personnel extraordinaire» (canonniers extraordinaires, pionniers, charretiers, etc.) levés en grand nombre mais seulement pour une campagne déterminée.

L'artillerie que Charles VIII emmène pour son expédition de 1494 est la première d'Europe et l'Italien Paul Jove l'a ainsi évoquée dans une page célèbre: «L'artillerie, comptait plus de 36 pièces que des chevaux tiraient à une incroyable vitesse, sur tous les terrains, unis ou accidentés. Les plus grandes, en bronze, longues de huit pieds et pesant six milles livres étaient appelées canons; ils lançaient des boulets de fer par un tube aussi large que la tête d'un homme. Puis venaient les coulevrines, plus longues mais jetant des boulets plus petits par des ouvertures plus étroites. Ensuite les faucons, aux dimensions variées.

so $\mathrm{H}$. Dubled: Les frères Bureau et l'artillerie royale française (1437-1469), in École Nationale des Chartes, Positions des thèses 1946, pp. 33-37.

${ }^{51} \mathrm{Ph}$. Contamine: L'artillerie royale française... (cf. note 14), surtout pp. 221-223. 
Les plus petits utilisaient des projectiles de la grosseur d'une orange. Toutes ces pièces étaient fixées aux affûts par deux épais crampons; pour régler le tir et pointer, des anses permettaient de les soulever. Les petites pièces étaient montées sur deux roues, les plus grandes sur quatre, celles de derrière pouvant être enlevées si on voulait accélerer le train ou l'arrêter...» ${ }^{52}$.

$$
* * *
$$

Comme il y a loin des canons primitifs figurés dans les miniatures des ouvrages de Walter de Milmete à ceux décrits par Paul Jove! Les perfectionnements de tout ordres ainsi que la place occupée par l'artillerie dans l'armée lors de la campagne d'Artois entreprise par Louis XI ${ }^{53}$, puis dans celles de Bretagne et d'Italie faites par son fils ${ }^{54}$, montrent clairement les progrès réalisés en moins de deux siècles.

p. 221.

52 Cité d'après Ph. Contamine: L'artillerie royale française... (cf. note 14),

${ }_{53}$ Ed. Perroy: L'artillerie de Louis XI (cf. note 15).

${ }^{54} \mathrm{PH}$. Contamine: L'artillerie royale française... (cf. note 14). 